\title{
Radial distribution function for hard spheres in fractal dimensions: A heuristic approximation
}

\author{
Andrés Santos* \\ Departamento de Física and Instituto de Computación Científica Avanzada (ICCAEx), \\ Universidad de Extremadura, Badajoz, E-06071, Spain \\ Mariano López de Haro ${ }^{\dagger}$ \\ Instituto de Energías Renovables, Universidad Nacional Autónoma de México (U.N.A.M.), Temixco, Morelos 62580, Mexico
}

(Dated: June 20, 2016)

\begin{abstract}
Analytic approximations for the radial distribution function, the structure factor, and the equation of state of hard-core fluids in fractal dimension $d(1 \leq d \leq 3)$ are developed as heuristic interpolations from the knowledge of the exact and Percus-Yevick results for the hard-rod and hard-sphere fluids, respectively. In order to assess their value, such approximate results are compared with those of recent Monte Carlo simulations and numerical solutions of the Percus-Yevick equation for a fractal dimension [M. Heinen et al., Phys. Rev. Lett. 115, 097801 (2015)], a good agreement being observed.
\end{abstract}

\section{INTRODUCTION}

The so-called classical fluids can, to a good approximation, be modeled and treated by the methods of classical statistical mechanics. Among these systems, the relatively simple hard-core models (hard rods, disks, spheres, and hyperspheres) have played a very important role in laying the foundations of a solid theoretical framework for dealing with the thermodynamic and structural properties of real fluids. Among their assets, it should be pointed out that all hard-core fluids share similar properties, including the existence of a fluid-solid phase transition (except in the one-dimensional case) that occurs at a packing fraction that becomes smaller as the dimensionality $d$ is increased. Thus, increasing the dimensionality of these hard-core fluids leads in general to simpler mathematical formulations to describe similar phenomenology [1]. On the other hand, while real fluids in the bulk are three-dimensional, once they are confined their effective dimension becomes $d=2$ or $d=1$. It is well known that confinement has a substantial influence on the thermodynamic and structural properties of fluids, a subject that has been profusely dealt with in the literature (see, for instance, Refs. 2-4 and references therein).

On the other hand, it is only very recently that Heinen et al. [5] have addressed the problem of formulating a theory for the fractal analog of the simplest classical generic model fluid in integer dimensions, namely, the hard-core fluid in fractal dimensions between $d=1$ and 3 . In their model, they consider fractal particles in a fractal configuration space, both of the same noninteger dimension. Such a generic model of fractal liquids can describe, for instance, microphase separated binary liquids in porous media and highly branched liquid droplets confined to a fractal polymer backbone in a gel. Heinen et al. [5] performed Monte Carlo (MC) simulations of fractal hard

\footnotetext{
* andres@unex.es; http://www.unex.es/eweb/fisteor/andres/Cvitae/

$\dagger$ malopez@unam.mx; http://xml.ier.unam.mx/xml/tc/ft/mlh/
}

"spheres" lying on a near-critical percolating lattice cluster. In this context, a fractal sphere of "diameter" $\sigma$ is defined by those lattice sites at a chemical distance (taxicab metric) from the sphere center site smaller than $\frac{1}{2} \sigma$. Moreover, Heinen et al. were able to (numerically) generalize the solution to the Ornstein-Zernike equation with the Percus-Yevick (PY) closure to noninteger dimensions. In that solution, the corresponding radial distribution function (RDF) and thermodynamic properties represent the analytic continuations of the standard PY theory with respect to the dimension.

The aim of this paper is to develop an analytic approximation for the RDF $g^{(d)}(r, \phi)$ of hard-core fluids in fractal dimension $d$ (with $1 \leq d \leq 3$ ), where $r$ is the distance (in the non-Euclidian fractal space) and $\phi \equiv\left[(\pi / 4)^{d / 2} / \Gamma(1+d / 2)\right] \rho \sigma^{d}$ is the packing fraction (with $\sigma$ the hard-core diameter and $\rho$ the number density). Following a heuristic approach similar to the one used to derive the RDF of a hard-disk fluid $(d=2)$ [6], our approximation will be constructed simply as an interpolation between the exact $\operatorname{RDF} g^{(1)}(r, \phi)$ at $d=1$ [7-12] and the PY solution $g^{(3)}(r, \phi)$ at $d=3[10,11,13-17]$, with suitably scaled packing fractions. The determination of the two scaling parameters requires the independent proposal of an analytic approximate expression for the contact value $g_{\text {cont }}^{(d)}(\phi) \equiv g^{(d)}\left(\sigma^{+}, \phi\right)$, and hence for the virial equation of state of the fractal fluid, and this represents an extra bonus of our heuristic approach. As we will see, despite the simplicity of the theory, the results agree fairly well with both MC simulations and PY numerical results obtained in Ref. [5] at $d=1.67659$.

The paper is organized as follows. In Sec. II, we propose the explicit expressions for $g_{\text {cont }}^{(d)}(\phi)$ and $g^{(d)}(r, \phi)$, and derive the compressibility factor $Z^{(d)}(\phi)$ that follows from both the virial and compressibility routes to the equation of state. In Sec. III, we perform an analysis of the behavior of the third and fourth virial coefficients that follow from our formulation, while our theoretical results for the compressibility factor, the RDF, and the structure factor are compared in Sec. IV with those of the 
generalized PY theory and the MC data presented in Ref. 5. The paper concludes in Sec. $\mathrm{V}$ with some concluding remarks.

\section{RADIAL DISTRIBUTION FUNCTION AND EQUATION OF STATE}

Due to their importance in our later development, before constructing the function $g^{(d)}(r, \phi)$, let us first consider two different quantities (one local and another one global) related to $g^{(d)}(r, \phi)$. On the one hand, we take the contact value $g_{\text {cont }}^{(d)}(\phi)$ defined above. The exact and PY expressions for this quantity in the cases $d=1$ and $d=3$ are, respectively,

$$
\begin{gathered}
g_{\text {cont }}^{(1)}(\phi)=\frac{1}{1-\phi}, \\
g_{\text {cont }}^{(3)}(\phi)=\frac{1+\phi / 2}{(1-\phi)^{2}} .
\end{gathered}
$$

We also note that the scaled-particle theory (SPT) approximation $[18,19]$ for $d=2$ has the form

$$
g_{\mathrm{cont}}^{(2)}(\phi)=\frac{1-a^{(2)} \phi}{(1-\phi)^{2}}
$$

with $a^{(2)}=\frac{1}{2}$. A more accurate fractional value $a^{(2)}=$ $\frac{7}{16}=0.4375$ was proposed by Henderson [20], who also noticed that the value $a^{(2)}=2 \sqrt{3} / \pi-2 / 3 \simeq 0.436$ guarantees that Eq. (2.2) reproduces the exact third virial coefficient of hard disks. Here we will adopt the latter value for $a^{(2)}$.

In view of Eqs. (2.1) and (2.2), it is suggestive to construct a simple generalization for $1 \leq d \leq 3$ as

$$
g_{\text {cont }}^{(d)}(\phi)=\frac{1-a^{(d)} \phi}{(1-\phi)^{2}} .
$$

The density-independent coefficient $a^{(d)}$ can be constructed as a quadratic polynomial in $d$ with coefficients such that $a^{(1)}=1, a^{(2)}=2 \sqrt{3} / \pi-2 / 3$, and $a^{(3)}=-\frac{1}{2}$. The resulting expression is

$$
a^{(d)}=\frac{1}{4}(5-d)(2-d)+(3-d)(d-1) a^{(2)} .
$$

Next, we consider a convenient global quantity related to $g^{(d)}(r, \phi)$, namely the first moment of the total correlation function, $h^{(d)}(r, \phi)=g^{(d)}(r, \phi)-1$, defined as

$$
H^{(d)}(\phi)=-\sigma^{-2} \int_{0}^{\infty} \mathrm{d} r r h^{(d)}(r, \phi)
$$

The exact and PY results for this quantity corresponding to $d=1$ and $d=3$ are [6], respectively,

$$
H^{(1)}(\phi)=\frac{1}{2}-\frac{2}{3} \phi+\frac{1}{4} \phi^{2},
$$

$$
H^{(3)}(\phi)=\frac{\frac{1}{2}-\frac{1}{20} \phi(2-\phi)}{1+2 \phi} .
$$

Note that the knowledge of $g_{\text {cont }}^{(d)}(\phi)$ determines the equation of state via the virial route as $[11,16]$

$$
Z_{\mathrm{vir}}^{(d)}(\phi)=1+2^{d-1} \phi g_{\mathrm{cont}}^{(d)}(\phi)
$$

where $Z^{(d)} \equiv p / \rho k_{B} T$ is the so-called compressibility factor $\left(p, k_{B}\right.$, and $T$ being the pressure, Boltzmann constant, and absolute temperature, respectively). On the other hand, the compressibility equation of state may be derived from the following relations $[11,16]$ :

$$
\begin{gathered}
\chi^{(d)}(\phi) \equiv\left[\frac{\partial \phi Z^{(d)}(\phi)}{\partial \phi}\right]^{-1}=1+\rho \int \mathrm{d} \mathbf{r} h^{(d)}(r, \phi) \\
=1+d 2^{d} \phi \sigma^{-d} \int_{0}^{\infty} \mathrm{d} r r^{d-1} h^{(d)}(r, \phi), \\
Z_{\text {comp }}^{(d)}(\phi)=\int_{0}^{1} \frac{\mathrm{d} t}{\chi^{(d)}(\phi t)}
\end{gathered}
$$

In particular, in the two-dimensional case $(d=2)$ the moment $H^{(2)}$ is directly related to the isothermal compressibility:

$$
\chi^{(2)}(\phi)=1-8 \phi H^{(2)}(\phi)
$$

Imposing thermodynamic consistency with Eqs. (2.2), (2.6), and (2.8), one obtains

$$
H^{(2)}(\phi)=\frac{\frac{1}{2}-\frac{1}{4} a^{(2)} \phi(3-\phi)}{1+\phi+\left[1-2 a^{(2)}\right] \phi^{2}(3-\phi)} .
$$

The simplest common structure of Eqs. (2.5) and (2.9) can be seen to consist in the ratio between a quadratic and a cubic function of $\phi$. Analogously to what we did in the case of the generalization (2.3), we keep such a structure for all $1 \leq d \leq 3$ with density-independent coefficients expressed as quadratic functions of $d$. The result is

$$
H^{(d)}(\phi)=\frac{\frac{1}{2}-A^{(d)} \phi+C^{(d)} \phi^{2}}{1+(d-1) \phi\left\{1+(3-d)\left[1-2 a^{(2)}\right] \phi(3-\phi)\right\}},
$$

with

$$
\begin{aligned}
& A^{(d)}=\frac{1}{60}(2-d)(63-23 d)+\frac{3}{4}(d-1)(3-d) a^{(2)} \\
& C^{(d)}=\frac{1}{20}(2-d)(8-3 d)+\frac{1}{4}(d-1)(3-d) a^{(2)}
\end{aligned}
$$

Now we turn to the construction of a function $g^{(d)}(r, \phi)$ that, apart from reducing to $g^{(1)}(r, \phi)$ and $g^{(3)}(r, \phi)$ in the limits $d \rightarrow 1$ and $d \rightarrow 3$, respectively, is consistent 
with Eqs. (2.3) and (2.10). Following the heuristic idea of Ref. 6, we assume the simple interpolation formula

$$
\begin{aligned}
g^{(d)}(r, \phi)= & \alpha^{(d)}(\phi) g^{(1)}\left(r, \lambda_{1}^{(d)}(\phi) \phi\right) \\
& +\left[1-\alpha^{(d)}(\phi)\right] g^{(3)}\left(r, \lambda_{3}^{(d)}(\phi) \phi\right),
\end{aligned}
$$

where the mixing parameter $\alpha^{(d)}(\phi)$ and the scaling factors $\lambda_{1}^{(d)}(\phi)$ and $\lambda_{3}^{(d)}(\phi)$ are functions of $\phi$ and $d$ to be determined. The exact and PY expressions for $g^{(1)}(r, \phi)$ and $g^{(3)}(r, \phi)$, respectively, are recalled in the Appendix.

To fix $\lambda_{1}^{(d)}(\phi)$ and $\lambda_{3}^{(d)}(\phi)$, and again as in Ref. 6, we impose consistency with (2.3) regardless of the choice of the mixing parameter $\alpha^{(d)}$. This implies that

$$
g_{\text {cont }}^{(d)}(\phi)=g_{\text {cont }}^{(1)}\left(\lambda_{1}^{(d)}(\phi) \phi\right)=g_{\text {cont }}^{(3)}\left(\lambda_{3}^{(d)}(\phi) \phi\right) \text {. }
$$

The scaling factors $\lambda_{1}^{(d)}(\phi)$ and $\lambda_{3}^{(d)}(\phi)$ have the following interpretation. Given a $d$-dimensional system at a packing fraction $\phi_{d}$, the effective packing fractions $\phi_{1}$ and $\phi_{3}$ of the reference one- and three-dimensional systems (i.e., those having the same contact value), are, respectively,

$$
\begin{aligned}
& \phi_{1}=\lambda_{1}^{(d)}\left(\phi_{d}\right) \phi_{d}, \\
& \phi_{3}=\lambda_{3}^{(d)}\left(\phi_{d}\right) \phi_{d} .
\end{aligned}
$$

In particular, setting $d=1$ in Eq. (2.13b) yields $\phi_{3}=$ $\lambda_{3}^{(1)}\left(\phi_{1}\right) \phi_{1}$. Combining this with Eq. (2.13b), we obtain $\lambda_{3}^{(1)}\left(\phi_{1}\right) \phi_{1}=\lambda_{3}^{(d)}\left(\phi_{d}\right) \phi_{d}$. Next, taking into account Eq. (2.13a) we derive the following consistency condition

$$
\lambda_{3}^{(1)}\left(\lambda_{1}^{(d)}\left(\phi_{d}\right) \phi_{d}\right)=\frac{\lambda_{3}^{(d)}\left(\phi_{d}\right)}{\lambda_{1}^{(d)}\left(\phi_{d}\right)} .
$$

Proceeding in a similar way, we also have

$$
\lambda_{1}^{(3)}\left(\lambda_{3}^{(d)}\left(\phi_{d}\right) \phi_{d}\right)=\frac{\lambda_{1}^{(d)}\left(\phi_{d}\right)}{\lambda_{3}^{(d)}\left(\phi_{d}\right)} .
$$

It can be easily checked that Eqs. (2.14) are indeed satisfied as a consequence of Eq. (2.12).

Using Eqs. (2.1), the solution to Eq. (2.12) is easily found to be

$$
\begin{gathered}
\lambda_{1}^{(d)}(\phi)=\frac{g_{\text {cont }}^{(d)}(\phi)-1}{\phi g_{\text {cont }}^{(d)}(\phi)} \\
\lambda_{3}^{(d)}(\phi)=\frac{1+4 g_{\text {cont }}^{(d)}(\phi)-\sqrt{1+24 g_{\text {cont }}^{(d)}(\phi)}}{4 \phi g_{\text {cont }}^{(d)}(\phi)} .
\end{gathered}
$$

Once the scaling parameters $\lambda_{1}^{(d)}(\phi)$ and $\lambda_{3}^{(d)}(\phi)$ are determined, we obtain the mixing parameter $\alpha^{(d)}(\phi)$ by imposing consistency between Eq. (2.11) and the moment (2.10):

$$
\alpha^{(d)}(\phi)=\frac{H^{(d)}(\phi)-H^{(3)}\left(\lambda_{3}^{(d)}(\phi) \phi\right)}{H^{(1)}\left(\lambda_{1}^{(d)}(\phi) \phi\right)-H^{(3)}\left(\lambda_{3}^{(d)}(\phi) \phi\right)} .
$$

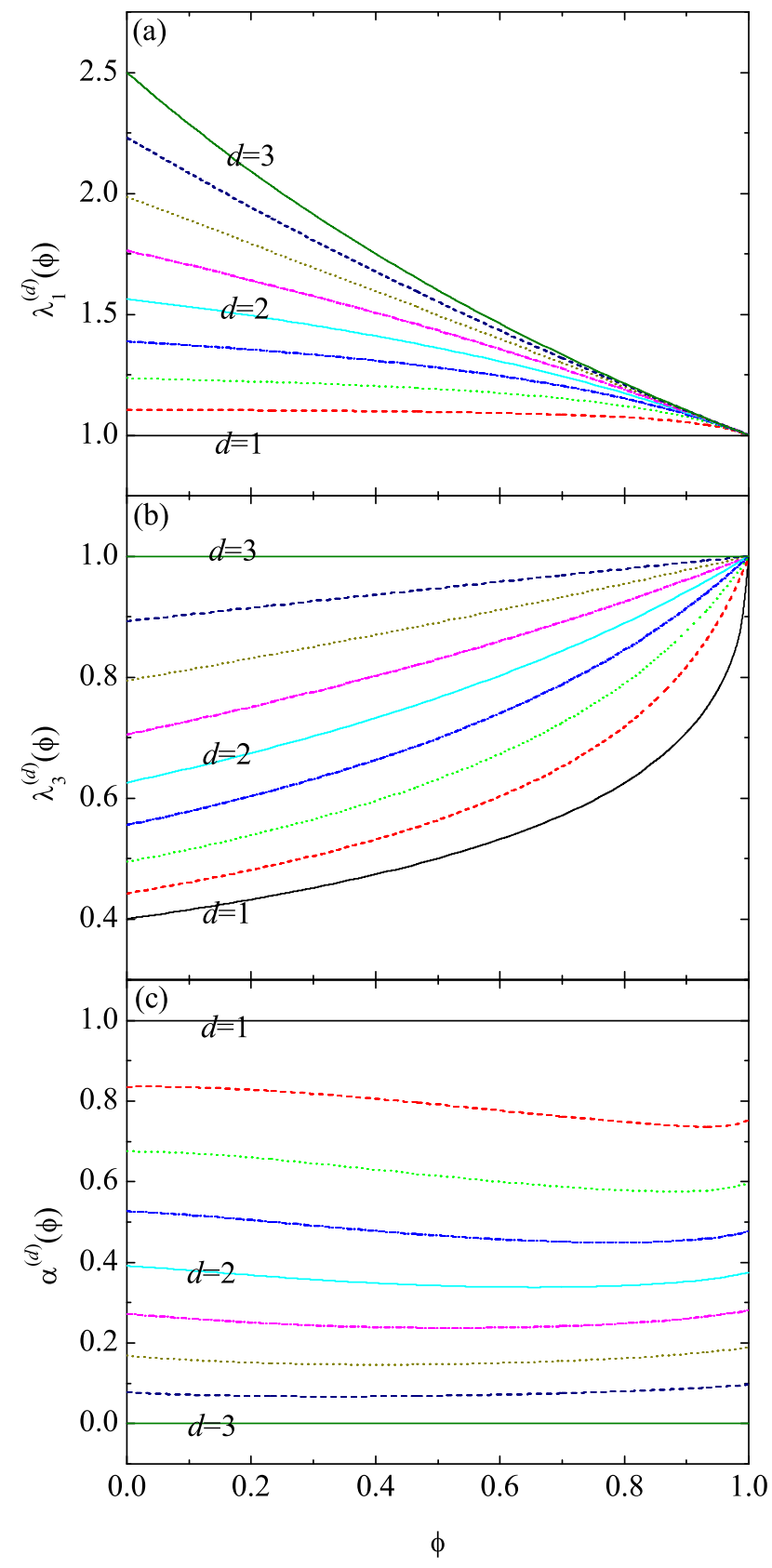

FIG. 1. Plot of (a) the scaling factor $\lambda_{1}^{(d)}(\phi)$, (b) the scaling factor $\lambda_{3}^{(d)}(\phi)$, and (c) the mixing parameter $\alpha^{(d)}(\phi)$ as functions of $\phi$ for $d=1,1.25,1.5,1.75,2,2.25,2.5,2.75,3$.

In summary, our proposal is defined by Eqs. (2.11) and $(2.15)-(2.17)$, with $g_{\text {cont }}^{(d)}(\phi)$ and $H^{(d)}(\phi)$ being given by Eqs. (2.3) and (2.10), respectively. In turn, the compressibility factor $Z^{(d)}(\phi)$ can be obtained either analytically from the virial route $(2.6)$, with the contact value $g_{\text {cont }}^{(d)}(\phi)$ given by Eqs. (2.3), or numerically from the compressibility route (2.7). By construction, the approximation (2.11) reduces to the exact and PY results in the limits $d \rightarrow 1$ and $d \rightarrow 3$, respectively, and is consistent (via both the virial and compressibility routes) with Hender- 
son's equation of state [see Eqs. (2.2) and (2.9)] in the limit $d \rightarrow 2$.

Note that the same method could still be applied by prescribing for $g^{(3)}(r, \phi)$ a RDF thermodynamically consistent and more accurate than the PY one, such as the rational-function approximation [11, 21-23], and/or by enforcing an equation of state for hard-disk fluids different from Henderson's. For simplicity, however, we keep the approximation (2.11) as formulated above [24].

Figure 1 shows $\lambda_{1}^{(d)}(\phi), \quad \lambda_{3}^{(d)}(\phi)$, and $\alpha^{(d)}(\phi)$ as functions of $\phi$ for the representative values $d=$ $1,1.25, \ldots, 2,2.25, \ldots, 3$. As expected on physical grounds, $\lambda_{1}^{(d)}(\phi)$ and $\lambda_{3}^{(d)}(\phi)$ are, respectively, monotonically decreasing and increasing functions of $\phi$. On the other hand, $\alpha^{(d)}(\phi)$ is very weakly dependent on $\phi$. Obviously, $\lambda_{1}^{(1)}(\phi)=1, \lambda_{3}^{(3)}(\phi)=1, \alpha^{(1)}(\phi)=1$, and $\alpha^{(3)}(\phi)=0$.

Before closing this section, let us also consider another structural property, namely the structure factor

$$
S^{(d)}(k, \phi)=1+\rho \widetilde{h}^{(d)}(k, \phi) .
$$

Here, the Fourier transform of the total correlation function is [5]

$$
\begin{aligned}
\widetilde{h}^{(d)}(k, \phi) & =\int \mathrm{d} \mathbf{r} e^{i \mathbf{k} \cdot \mathbf{r}} h^{(d)}(r, \phi) \\
& =\frac{(2 \pi)^{d / 2}}{k^{d / 2-1}} \int_{0}^{\infty} \mathrm{d} r r^{d / 2} h^{(d)}(r, \phi) J_{d / 2-1}(k r),
\end{aligned}
$$

where $J_{\nu}(x)$ is the Bessel function. Insertion of Eq. (2.11) yields

$$
\begin{aligned}
S^{(d)}(k, \phi)= & +\frac{\alpha^{(d)}(\phi)}{\lambda_{1}^{(d)}(\phi)} I_{1}^{(d)}\left(k, \lambda_{1}^{(d)}(\phi) \phi\right) \\
& +\frac{1-\alpha^{(d)}(\phi)}{\lambda_{3}^{(d)}(\phi)} I_{3}^{(d)}\left(k, \lambda_{3}^{(d)}(\phi) \phi\right),
\end{aligned}
$$

where we have called

$$
\begin{aligned}
I_{1,3}^{(d)}(k, \phi) \equiv & \frac{2^{3 d / 2} \Gamma(1+d / 2)}{k^{d / 2-1}} \sigma^{-d} \phi \\
& \times \int_{0}^{\infty} \mathrm{d} r r^{d / 2} h^{(1,3)}(r, \phi) J_{d / 2-1}(k r) .
\end{aligned}
$$

\section{LOW-DENSITY PROPERTIES}

In order to illustrate some of the consequences of our proposal, in this section we analyze its low-density predictions. To first order in density, one can write

$$
g^{(d)}(r, \phi)=\Theta(r-1)\left[1+\gamma^{(d)}(r) \phi+\mathcal{O}\left(\phi^{2}\right)\right],
$$

where, without loss of generality, henceforth we have set the value of the hard-core diameter to be $\sigma=1$ (i.e.,

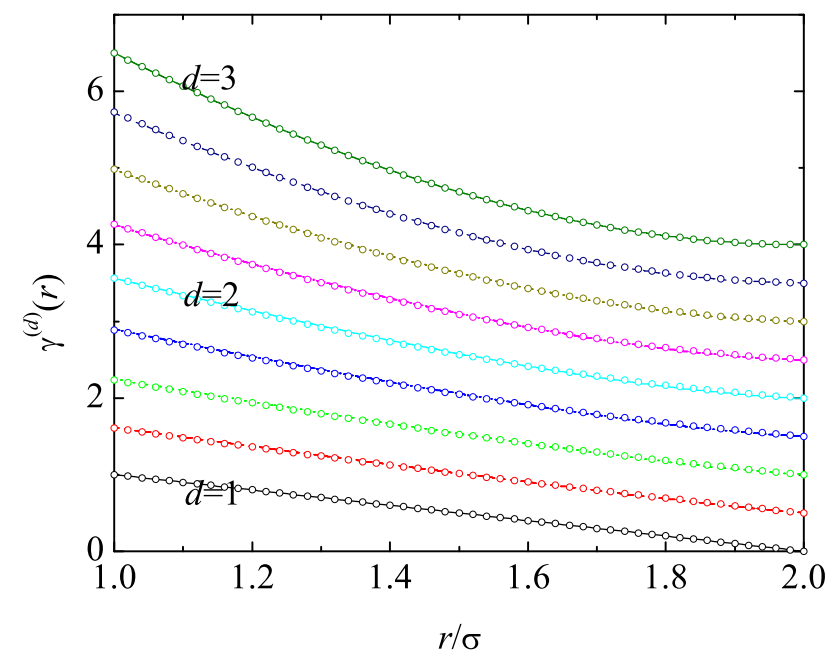

FIG. 2. Function $\gamma^{(d)}(r)$ for $d=$ $1,1.25,1.5,1.75,2,2.25,2.5,2.75,3$. The curves and the circles correspond to the exact function (3.2) and the approximation (3.5), respectively. Note that, for better visibility, the curves have been shifted vertically a distance $0,0.5, \ldots, 4$ for $d=1,1.25, \ldots, 3$, respectively.

distances are measured in units of the hard-core diameter) and $\Theta(x)$ is the Heaviside step function. The exact function $\gamma^{(d)}(r)$ is given by [25]

$$
\gamma_{\text {exact }}^{(d)}(r)=2^{d} \frac{B_{1-r^{2} / 4}\left(\frac{d+1}{2}, \frac{1}{2}\right)}{B\left(\frac{d+1}{2}, \frac{1}{2}\right)} \Theta(2-r),
$$

$B_{x}(a, b)$ and $B(a, b)$ being the incomplete and complete beta functions, respectively $[26,27]$. In particular,

$$
\begin{gathered}
\gamma^{(1)}(r)=(2-r) \Theta(2-r), \\
\gamma^{(3)}(r)=\frac{1}{2}(2-r)^{2}(4+r) \Theta(2-r) .
\end{gathered}
$$

On the other hand, use of the approximation (2.11) yields

$$
\gamma^{(d)}(r)=\alpha_{0}^{(d)} \lambda_{1,0}^{(d)} \gamma^{(1)}(r)+\left[1-\alpha_{0}^{(d)}\right] \lambda_{3,0}^{(d)} \gamma^{(3)}(r),
$$

where

$$
\begin{gathered}
\alpha_{0}^{(d)} \equiv \lim _{\phi \rightarrow 0} \alpha^{(d)}(\phi)=\frac{3}{34} \frac{50 A^{(d)}+22 a^{(d)}+25 d-69}{2-a^{(d)}} \\
\lambda_{1,0}^{(d)} \equiv \lim _{\phi \rightarrow 0} \lambda_{1}^{(d)}(\phi)=2-a^{(d)} \\
\lambda_{3,0}^{(d)} \equiv \lim _{\phi \rightarrow 0} \lambda_{3}^{(d)}(\phi)=\frac{2}{5}\left[2-a^{(d)}\right]
\end{gathered}
$$

As seen from Fig. 2, the values obtained from Eq. (3.5) are practically indistinguishable from the exact ones, Eq. (3.2). 


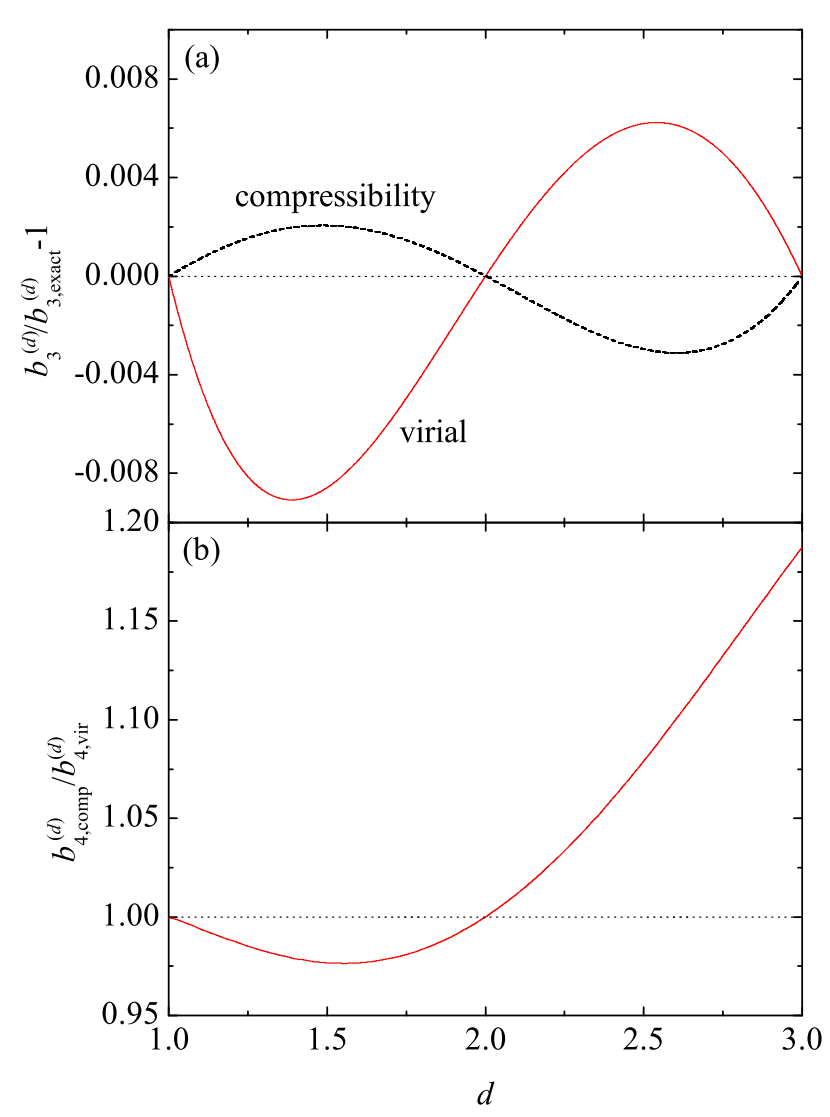

FIG. 3. (a) Relative differences $b_{3, \text { vir }}^{(d)} / b_{3 \text {,exact }}^{(d)}-1$ (solid line) and $b_{3, \text { comp }}^{(d)} / b_{3 \text {,exact }}^{(d)}-1$ (dashed line) versus $d$. (b) Ratio $b_{4, \mathrm{comp}}^{(d)} / b_{4, \mathrm{vir}}^{(d)}$ versus $d$.

Next, we consider the virial coefficients $b_{n}^{(d)}$ defined by the power series

$$
Z^{(d)}(\phi)=1+\sum_{n=2}^{\infty} b_{n}^{(d)} \phi^{n-1}
$$

As can be seen from Eqs. (2.6) and (3.1), the second virial coefficient is $b_{2}^{(d)}=2^{d-1}$. As for the third virial coefficient, its exact expression reads [25]

$$
b_{3, \text { exact }}^{(d)}=2^{2 d-1} \frac{B_{3 / 4}\left(\frac{d+1}{2}, \frac{1}{2}\right)}{B\left(\frac{d+1}{2}, \frac{1}{2}\right)} .
$$

On the other hand, according to the approximation (3.5), the coefficients stemming from the virial route (2.6) and from the compressibility route $(2.7)$ are

$$
\begin{gathered}
b_{3, \mathrm{vir}}^{(d)}=2^{d-1}\left[2-a^{(d)}\right] \\
b_{3, \mathrm{comp}}^{(d)}=\frac{2^{d}}{3}\left\{2^{d}-\alpha_{0}^{(d)} \lambda_{1,0}^{(d)} \frac{2^{d+1}-d-2}{d+1}-\left[1-\alpha_{0}^{(d)}\right]\right. \\
\left.\times \lambda_{3,0}^{(d)} \frac{3 \times 2^{d+4}-5 d^{2}-29 d-48}{2(d+1)(d+3)}\right\}
\end{gathered}
$$

The relative deviations of $b_{3 \text {,vir }}^{(d)}$ and $b_{3, \text { comp }}^{(d)}$ from $b_{3 \text {,exact }}^{(d)}$ are plotted in Fig. 3(a). The prescriptions (3.9) are exact at $d=1,2$, and 3 . One can see that the highest deviations of $b_{3, \text { vir }}^{(d)}$ from $b_{3 \text {,exact }}^{(d)}$ are $-0.9 \%(d=1.389)$ and $+0.6 \%(d=2.539)$. In the case of $b_{3 \text {,comp }}^{(d)}$ the highest deviations are $+0.2 \%(d=1.485)$ and $-0.3 \%(d=2.603)$.

In respect to the fourth virial coefficient $b_{4}^{(d)}$, although it is exactly known for integer values of $d$ [28, 29], an analytic continuation to fractional $d$ is, to the best of our knowledge, not available. On the other hand, the fourth virial coefficients that follow from the virial route, $b_{4, \text { vir }}^{(d)}$, and from the compressibility route, $b_{4, \text { comp }}^{(d)}$, can easily be derived from Eqs. (2.6) and (2.7), respectively. The ratio $b_{4, \text { comp }}^{(d)} / b_{4, \text { vir }}^{(d)}$ is plotted in Fig. $3(\mathrm{~b})$. We observe that, in contrast to what happens in the case of $b_{3}^{(d)}$, one has $b_{4, \text { comp }}^{(d)}<b_{4 \text {,vir }}^{(d)}$ for $1<d<2$ and $b_{4 \text {, comp }}^{(d)}>b_{4, \text { vir }}^{(d)}$ for $2<d \leq 3$. Obviously, the PY value $b_{4, \text { comp }}^{(3)} / b_{4, \text { vir }}^{(3)}=$ $19 / 16=1.1875$ is recovered at $d=3$.

\section{RESULTS AT FINITE DENSITY}

Once we have studied the main properties of our proposal in the low-density regime, we turn our attention to the more important case of finite densities.

We first discuss the compressibility factor. Figure 4 compares $Z_{\text {vir }}^{(d)}(\phi)$ and $Z_{\text {comp }}^{(d)}(\phi)$, as predicted by our heuristic approximation, with $Z_{\text {vir }}^{(d)}(\phi)$, as obtained from the numerical solution of the PY integral equation [5, 31]. It can be observed that the simple equation of state defined by the combination of Eqs. (2.3) and (2.6) gives values close to (but slightly larger than) those obtained from the PY equation. Of course, both approximations coincide, by construction, at $d=3$. As for the compressibility-route function $Z_{\text {comp }}^{(d)}(\phi)$ obtained from our approach, it practically coincides with $Z_{\text {vir }}^{(d)}(\phi)$ up to $Z^{(d)} \approx 4$. Thereafter, one has $Z_{\text {comp }}^{(d)}(\phi)<Z_{\text {vir }}^{(d)}(\phi)$ for $1<d<2$ and $Z_{\text {comp }}^{(d)}(\phi)>Z_{\text {vir }}^{(d)}(\phi)$ for $2<d \leq 3$, in qualitative agreement with Fig. $3(\mathrm{~b})$.

To analyze the degree of thermodynamic inconsistency between $Z_{\text {comp }}^{(d)}(\phi)$ and $Z_{\text {vir }}^{(d)}(\phi)$ with more detail, we choose, for each $d$, a reference packing fraction $\phi_{\text {ref }}$ such that $Z_{\text {vir }}^{(d)}\left(\phi_{\text {ref }}\right)=Z_{\text {ref }}$, where $Z_{\text {ref }}$ is a certain common value. Figure 5 shows the curves corresponding to $Z_{\text {ref }}=6$ and $Z_{\text {ref }}=10$. As can be observed, the inconsistency is typically smaller for $1<d<2$ than for $2<d<3$. In the latter interval, the highest discrepancy does not correspond to $d=3$ but to $d \approx 2.7$. Taking into account the known behavior at $d=3$, it is suggestive to speculate that the correct value of the compressibility factor lies approximately between $Z_{\text {comp }}^{(d)}(\phi)$ and $Z_{\text {vir }}^{(d)}(\phi)$, perhaps closer to the former than to the latter. This conjecture is supported by Fig. 3(a). 

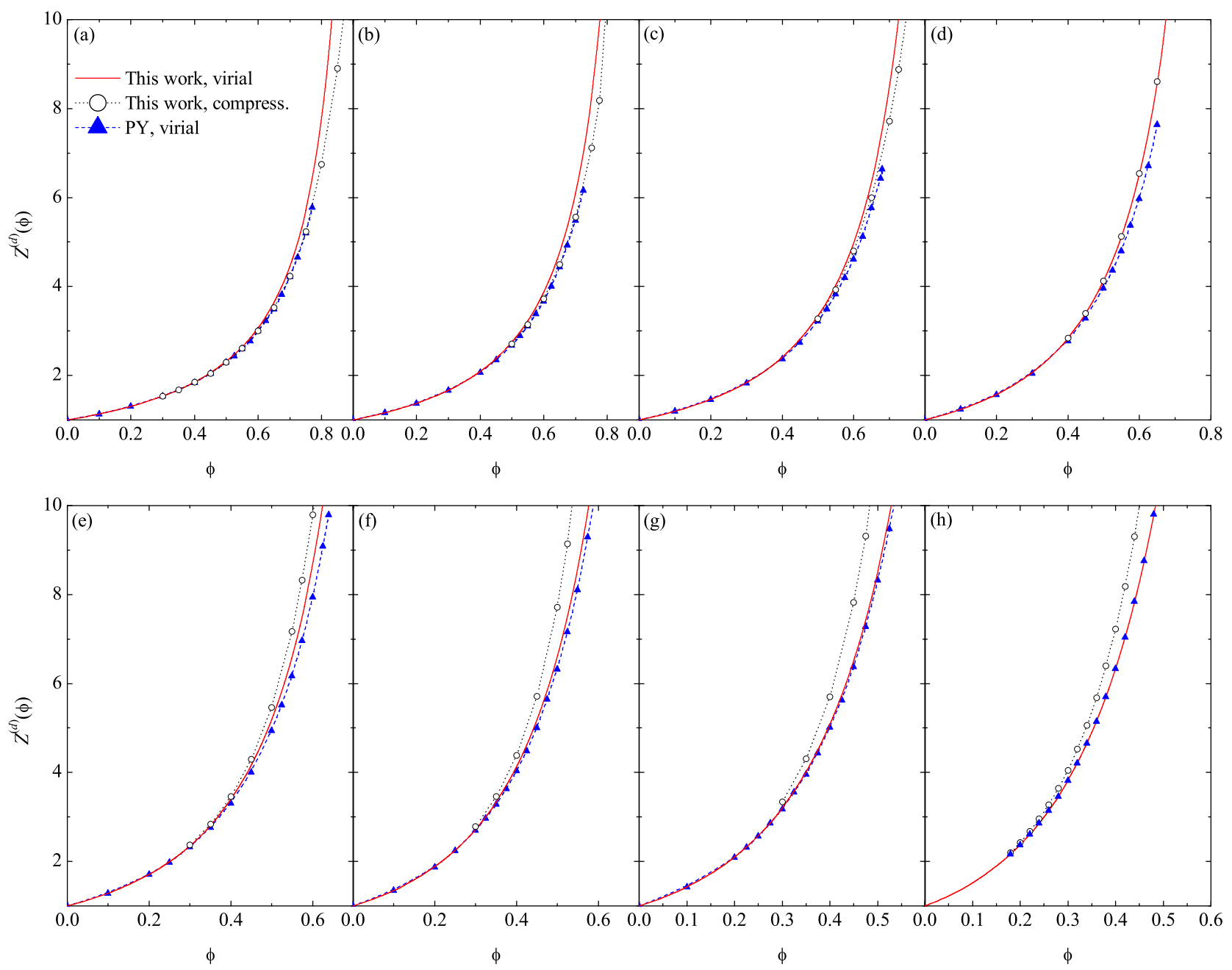

FIG. 4. Plot of the compressibility factor $Z^{(d)}(\phi)$ for (a) $d=1.25$, (b) $d=1.5$, (c) $d=1.75$, (d) $d=2$, (e) 2.25 , (f) $d=2.5$, (g) $d=2.75$, and $(\mathrm{h}) d=3$. The curves without symbols and the curves with circles represent the results obtained from our approach through the virial and compressibility routes, respectively. The curves with triangles correspond to $Z_{\mathrm{vir}}^{(d)}(\phi)$ as obtained from the numerical solution of the PY integral equation [5].

Let us consider now the RDF. Figure 6 compares our simple approximation (2.11) against MC simulations and numerical solutions of the PY equation for the fractal dimensionality $d=1.67659$ and two packing fractions [5]. We observe that at the lowest density $(\phi=0.266)$ the results obtained from the heuristic approach (2.11) are practically indistinguishable from the PY values, both describing fairly well the MC data. At a higher density $(\phi=0.487)$, however, small differences are visible, especially around the first minimum and the second maximum. Although the noise in the MC data does not allow for a definite conclusion, a slightly better performance of Eq. (2.11) seems to be present.

The good agreement observed in Fig. 6 between our simple approach and the much more demanding numerical solution of the PY integral equation [31] is nicely confirmed by Fig. 7 at the level of the structure factor. Both theories yield practically indistinguishable results at $\phi=0.266$. At $\phi=0.487$, the only visible differences appear around the first peak and the first minimum (different from the one at $k=0)$ of $S^{(d)}(k, \phi)$, where the values obtained from Eq. (2.20) are slightly smaller than the PY ones.

\section{CONCLUDING REMARKS}

Motivated by the recent work of Heinen et al. [5], in this paper we have constructed a heuristic approximation for the $\mathrm{RDF} g^{(d)}(r, \phi)$ and its associated structure factor $S^{(d)}(k, \phi)$ of hard-core fluids in fractal dimension $(1 \leq d \leq 3)$ [cf. Eqs. (2.11) and (2.20)], which is exact for $d=1$ and reduces to the PY result for $d=3$. Also, using $g^{(d)}(r, \phi)$, approximations for the compressibility factor $Z^{(d)}(\phi)$ of such fluids have been derived either analytically from the virial route [cf. Eq. (2.6), with the contact value $g_{\text {cont }}^{(d)}(\phi)$ given by Eqs. (2.3)], or numeri- 


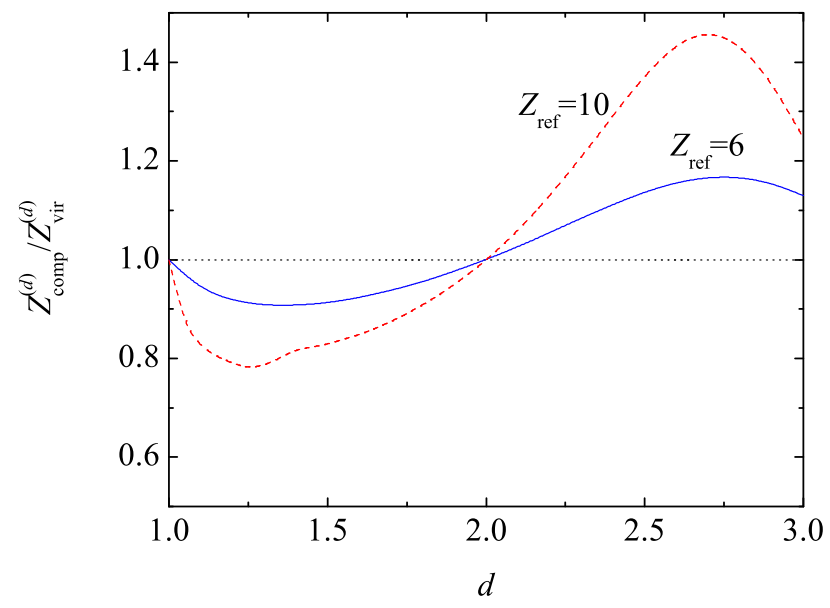

FIG. 5. Plot of the ratio $Z_{\mathrm{comp}}^{(d)} / Z_{\mathrm{vir}}^{(d)}$ as a function of $d$. For each value of $d$, a packing fraction $\phi_{\text {ref }}$ is chosen such that $Z_{\text {vir }}^{(d)}\left(\phi_{\text {ref }}\right)=Z_{\text {ref }}$, where $Z_{\text {ref }}$ is a common value. The two curves correspond to $Z_{\text {ref }}=6$ and $Z_{\text {ref }}=10$.

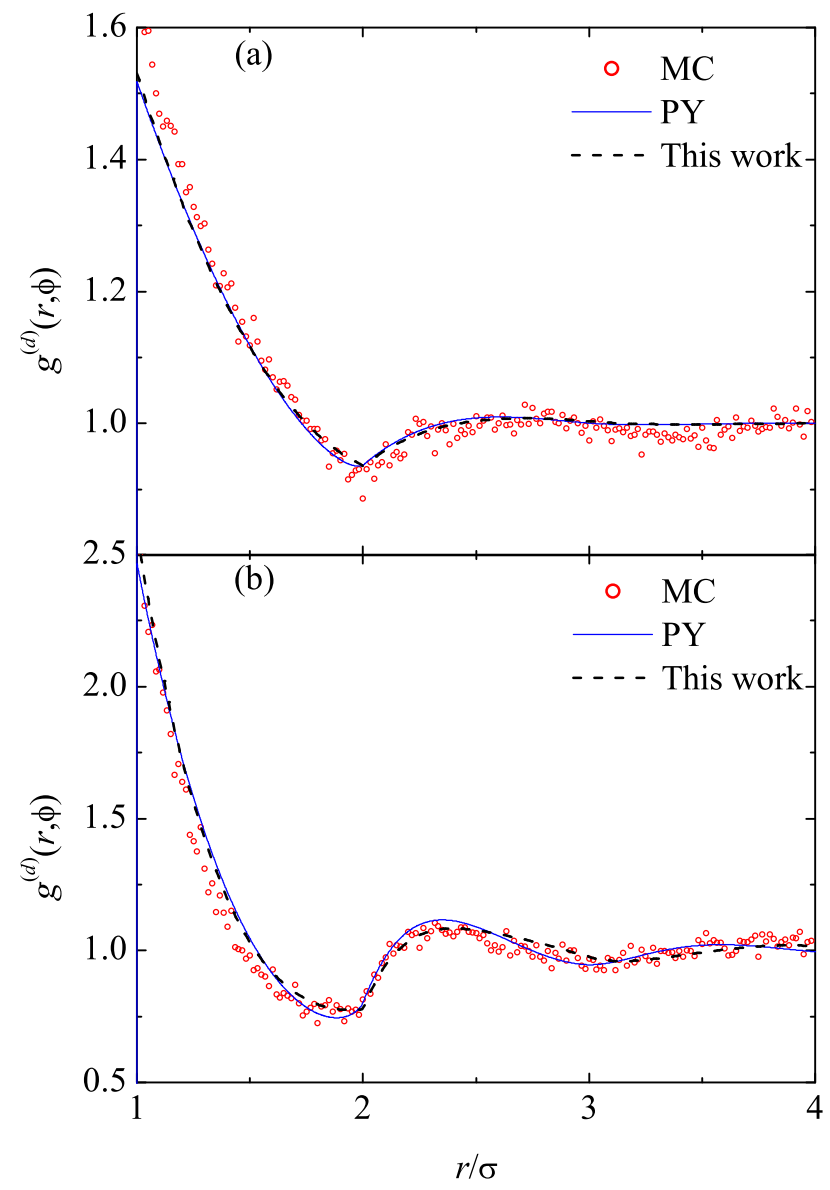

FIG. 6. Plot of $g^{(d)}(r, \phi)$ at (a) $\phi=0.266$ and (b) $\phi=0.487$ for $d=1.67659$. The symbols correspond to MC simulations [5], while the solid and dashed curves represent the numerical solution of the PY integral equation [5] and the analytical approximation (2.11), respectively.

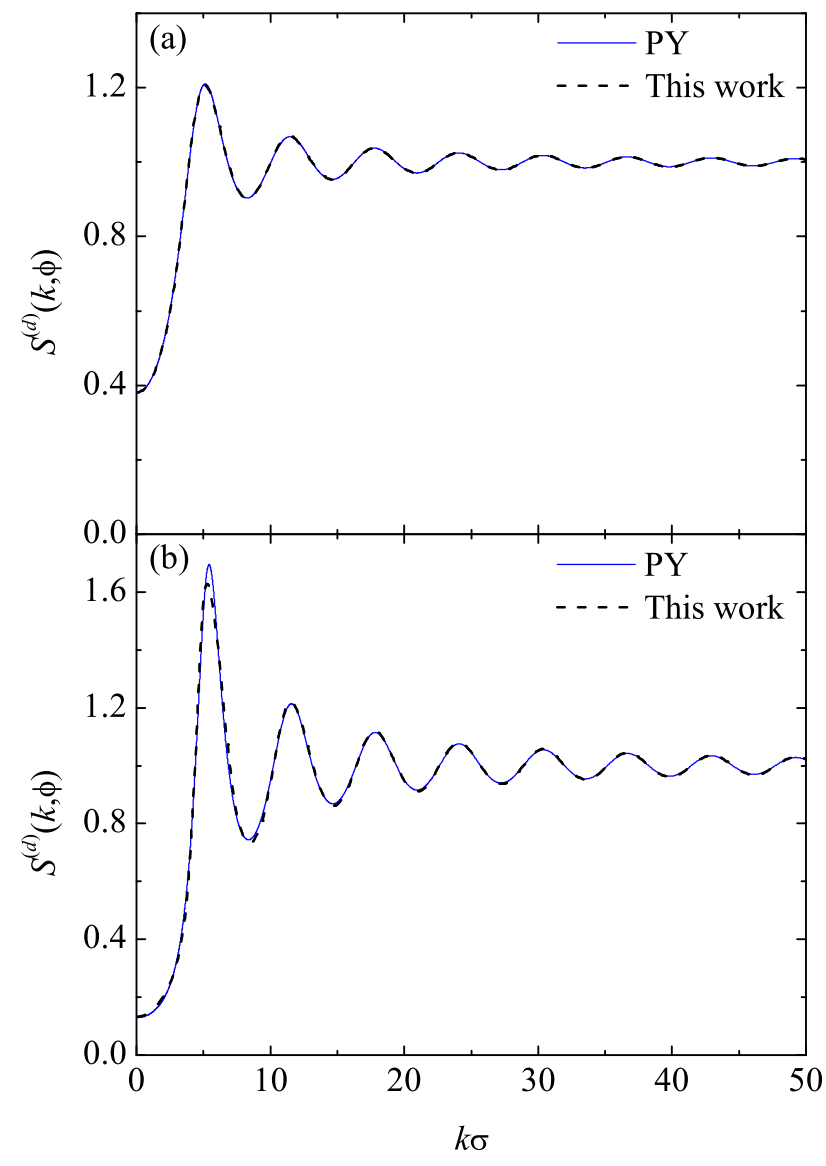

FIG. 7. Plot of $S^{(d)}(k, \phi)$ at (a) $\phi=0.266$ and (b) $\phi=0.487$ for $d=1.67659$. The solid and dashed curves represent the numerical solution of the PY integral equation [30] and the approximation (2.20), respectively.

cally from the compressibility route [cf. Eqs. (2.7)]. A noteworthy aspect is that, by construction, the virial and compressibility routes are thermodynamically consistent in the cases $d=1$ (exact solution) and $d=2$ (Henderson's equation of state), while the two well-known PY equations of state are recovered in the case $d=3$. Moreover, we found that the approximate RDF to first order in density is practically indistinguishable from the exact one and the relative deviations of the third virial coefficients $b_{3 \text {,vir }}^{(d)}$ and $b_{3, \text { comp }}^{(d)}$ (which are exact for $d=1,2$, and 3 ) from $b_{3 \text {,exact }}^{(d)}$ are below $\pm 1 \%$. Further, the comparison between our results for $g^{(d)}(r, \phi)$ and the available MC and PY results [5] for hard-core fluids in fractal dimension $(d=1.67659)$, indicates that our proposal is much simpler and as accurate as the PY theory, and in some instances it shows a slightly better agreement with the MC data than the PY theory.

As said in Sec. II, it would be possible to improve the accuracy of our proposal (2.11) by employing for the three-dimensional RDF $g^{(3)}(r, \phi)$ a more consistent and reliable form $[11,21-23]$ than the PY one. While this alternative implementation is not expected to have a rel- 
evant impact for fractal dimensions $1<d<2$, it might be interesting to explore it in the range $2<d<3$, provided computer simulations and/or numerical solutions of integral equations were available. Another possible extension of our work in the same range of values for the fractal dimension is the case of mixtures, where again the exact solution for $d=1$ and the PY solution for $d=3$ are known [9, 11, 32].

Although our proposal has been devised explicitly for $1<d<3$, in principle one could attempt to extrapolate it for fractal dimensions slightly higher than $d=3$. Of course it should be expected that the quality of the agreement will deteriorate as $d$ increases, especially beyond the low-density regime. As an illustration that this expectation is fulfilled, we find that, for $d=4$, our approximation yields $b_{3, \text { vir }}^{(4)}=30.4638, b_{3, \text { comp }}^{(4)}=34.3734$, $b_{4, \mathrm{vir}}^{(4)}=52.9276$, and $b_{4, \mathrm{comp}}^{(4)}=64.3768$, while the exact results are $b_{3 \text {,exact }}^{(4)}=32.4058$ and $b_{4 \text {,exact }}^{(4)}=77.7452$. In fact, for $3<d<5$, a much better approximation should be obtained by following a similar interpolation procedure but using the known PY results for $g^{(3)}(r, \phi)$ and $g^{(5)}(r, \phi)[33-35]$ instead of $g^{(1)}(r, \phi)$ and $g^{(3)}(r, \phi)$, as done here. Finally, as far as possible generalizations to particle interactions other than hard-core within the range $1<d<3$ are concerned, the case that immediately comes to mind is the one corresponding to sticky hard spheres. In such a case the exact RDF is known for $d=1[11,36,37]$ and the PY result for $d=3$ is also available [11, 38, 39].

\section{ACKNOWLEDGMENTS}

We want to thank M. Heinen for supplying simulation MC data and numerical results of the PY theory, as well as for a fruitful exchange of correspondence. We also acknowledge the financial support of the Ministerio de Economía y Competitividad (Spain) through Grant No. FIS2013-42840-P and the Junta de Extremadura (Spain) through Grant No. GR15104 (partially financed by FEDER funds).

\section{Appendix: Radial distribution function of hard rods and hard spheres}

\section{Hard rods}

In the one-dimensional case $(d=1)$, the Laplace transform

$$
G^{(1)}(s, \phi)=\int_{0}^{\infty} \mathrm{d} r e^{-r s} g^{(1)}(r, \phi)
$$

is exactly given by $[9,11]$

$$
G^{(1)}(s, \phi)=\frac{1}{\phi} \frac{e^{-s}}{1+s(1-\phi) / \phi-e^{-s}},
$$

where again we have set $\sigma=1$ as length unit. Expanding $G^{(1)}(s, \phi)$ in powers of $e^{-s}$, it is easy to perform an inverse Laplace transform term by term to obtain

$$
\begin{aligned}
g^{(1)}(r, \phi)= & \frac{1}{\phi} \sum_{\ell=1}^{\infty}\left(\frac{\phi}{1-\phi}\right)^{\ell} \frac{(r-\ell)^{\ell-1}}{(\ell-1) !} \\
& \times e^{-(r-\ell) \phi /(1-\phi)} \Theta(r-\ell) .
\end{aligned}
$$

This representation can be truncated at $\ell=\ell_{c}$ if only the range $1 \leq r \leq \ell_{c}+1$ is relevant. On the other hand, the tail of the total correlation function is in principle necessary to complement (A.3) in the evaluation of the integrals in (2.7a) and (2.21). The asymptotic tail of $h^{(1)}(r, \phi)$ is [40]

$$
h^{(1)}(r, \phi) \approx K^{(1)}(\phi) e^{-\kappa^{(1)}(\phi) r} \cos \left[\omega^{(1)}(\phi) r+\delta^{(1)}(\phi)\right],
$$

where $s_{ \pm}=-\kappa^{(1)} \pm i \omega^{(1)}$ are the two complex conjugate poles of $G^{(1)}(s)$ with a real part closest to the origin and $\frac{1}{2} K^{(1)} e^{i \delta^{(1)}}$ is the associated residue. More explicitly, $\kappa^{(1)}$ and $\omega^{(1)}$ are roots of the coupled set of transcendental equations

$$
\begin{aligned}
1-\frac{1-\phi}{\phi} \kappa^{(1)} & =e^{\kappa^{(1)}} \cos \omega^{(1)} \\
-\frac{1-\phi}{\phi} \omega^{(1)} & =e^{\kappa^{(1)}} \sin \omega^{(1)}
\end{aligned}
$$

Once $s_{ \pm}=-\kappa^{(1)} \pm i \omega^{(1)}$ are known, the amplitude and phase are

$$
\begin{aligned}
K^{(1)} & =2\left|1+\frac{1-\phi}{\phi+(1-\phi) s_{ \pm}}\right|^{-1} \\
\delta^{(1)} & =-\arg \left[1+\frac{1-\phi}{\phi+(1-\phi) s_{ \pm}}\right] .
\end{aligned}
$$

\section{Hard spheres}

The PY solution for the three-dimensional case has a structure reminiscent of that of the one-dimensional case. First, the Laplace transform

$$
G^{(3)}(s, \phi)=\int_{0}^{\infty} \mathrm{d} r e^{-r s} r g^{(3)}(r, \phi)
$$

is introduced. The PY solution is then found to be [11, 14, 15]

$$
G^{(3)}(s, \phi)=s \frac{F(s, \phi) e^{-s}}{1+12 \phi F(s, \phi) e^{-s}},
$$

where

$$
F(s, \phi)=-\frac{1}{12 \phi} \frac{1+L_{1}(\phi) s}{1+S_{1}(\phi) s+S_{2}(\phi) s^{2}+S_{3}(\phi) s^{3}}
$$


with the coefficients

$$
\begin{aligned}
L_{1}(\phi) & =\frac{1+\phi / 2}{1+2 \phi}, \quad S_{1}(\phi)=-\frac{3}{2} \frac{\phi}{1+2 \phi}, \quad(\mathrm{A} . \\
S_{2}(\phi) & =-\frac{1}{2} \frac{1-\phi}{1+2 \phi}, \quad S_{3}(\phi)=-\frac{1}{12 \phi} \frac{(1-\phi)^{2}}{1+2 \phi} .
\end{aligned}
$$

Again, a formal expansion of Eq. (A.8) in powers of $e^{-s}$ allows one to write

$$
g^{(3)}(r, \phi)=\frac{1}{r} \sum_{\ell=1}^{\infty}(-12 \phi)^{\ell-1} \Psi_{\ell}(r-\ell, \phi) \Theta(r-\ell),
$$

where

$$
\begin{gathered}
\Psi_{\ell}(r, \phi)=\sum_{j=1}^{\ell} \frac{\sum_{i=1}^{3} a_{\ell j}^{(i)}(\phi) e^{s_{i}(\phi) r}}{(\ell-j) !(j-1) !} r^{\ell-j}, \\
a_{\ell j}^{(i)}(\phi)=\lim _{s \rightarrow s_{i}(\phi)}\left(\frac{\partial}{\partial s}\right)^{j-1}\left\{s\left[\left(s-s_{i}(\phi)\right) F(s, \phi)\right]^{\ell}\right\} .
\end{gathered}
$$

Here, $s_{i}(\phi),(i=1,2,3)$ are the three roots of the cubic equation $1+S_{1}(\phi) s+S_{2}(\phi) s^{2}+S_{3}(\phi) s^{3}=0$. As in the case of Eq. (A.3), the summation in Eq. (A.11) can be truncated at $\ell=\ell_{c}$ to obtain the RDF within the interval $1 \leq r \leq \ell_{c}+1$. This can be complemented by the asymptotic tail of the total correlation function $[40,41]$,

$$
h^{(3)}(r, \phi) \approx \frac{K^{(3)}(\phi)}{r} e^{-\kappa^{(3)}(\phi) r} \cos \left[\omega^{(3)}(\phi) r+\delta^{(3)}(\phi)\right],
$$

where $s_{ \pm}=-\kappa^{(3)} \pm i \omega^{(3)}$ are the two complex conjugate poles of $G^{(3)}(s)$ with a real part closest to the origin, $\frac{1}{2} K^{(3)} e^{i \delta^{(3)}}$ being the associated residue. Thus, the transcendental equation for $s_{ \pm}$is

$$
\left(1+L_{1} s_{ \pm}\right) e^{-s_{ \pm}}=1+S_{1} s_{ \pm}+S_{2} s_{ \pm}^{2}+S_{3} s_{ \pm}^{3},
$$

while the residue is

$$
\operatorname{Res}=\frac{s_{ \pm}\left(1+L_{1} s_{ \pm}\right) / 12 \phi}{L_{1}\left(s_{ \pm}-1\right)-1-e^{s_{ \pm}}\left(S_{1}+2 S_{2} s_{ \pm}+3 S_{3} s_{ \pm}^{2}\right)},
$$

so that

$$
K^{(3)}=2|\operatorname{Res}|, \quad \delta^{(3)}=\arg (\operatorname{Res}) .
$$

[1] P. Charbonneau, A. Ikeda, G. Parisi, and F. Zamponi, Phys. Rev. Lett. 107, 185702 (2011).

[2] H. Löwen, J. Phys.: Condens. Matter 21, 474203 (2009).

[3] T. Franosch, S. Lang, and R. Schilling, Phys. Rev. Lett. 109, 240601 (2012).

[4] T. Franosch, S. Lang, and R. Schilling, Phys. Rev. Lett. 110, 059901(E) (2013).

[5] M. Heinen, S. K. Schnyder, J. F. Brady, and H. Löwen, Phys. Rev. Lett. 115, 097801 (2015).

[6] S. B. Yuste and A. Santos, J. Chem. Phys. 99, 2020 (1993).

[7] K. F. Herzfeld and M. Goeppert-Mayer, J. Chem. Phys. 2, 38 (1934).

[8] L. Tonks, Phys. Rev. 50, 955 (1936).

[9] M. Heying and D. S. Corti, Fluid Phase Equil. 220, 85 (2004).

[10] A. Santos, in 5th Warsaw School of Statistical Physics, edited by B. Cichocki, M. Napiórkowski, and J. Piasecki (Warsaw University Press, Warsaw, 2014) http://arxiv.org/abs/1310.5578.

[11] A. Santos, A Concise Course on the Theory of Classical Liquids. Basics and Selected Topics, Lecture Notes in Physics, Vol. 923 (Springer, 2016).

[12] A. Santos, (2015), "Radial Distribution Functions for Nonadditive Hard-Rod Mixtures", Wolfram Demonstrations Project, http://demonstrations.wolfram.com/ RadialDistributionFunctionsForNonadditiveHardRodMix

[13] E. Thiele, J. Chem. Phys. 39, 474 (1963).

[14] M. S. Wertheim, Phys. Rev. Lett. 10, 321 (1963).

[15] M. S. Wertheim, J. Math. Phys. 5, 643 (1964).
[16] J.-P. Hansen and I. R. McDonald, Theory of Simple Liquids, 3rd ed. (Academic, London, 2006).

[17] A. Santos, (2013), "Radial Distribution Function for Hard Spheres", Wolfram Demonstrations Project, http://demonstrations.wolfram.com/ RadialDistributionFunctionForHardSpheres/.

[18] H. Reiss, H. L. Frisch, and J. L. Lebowitz, J. Chem. Phys. 31, 369 (1959).

[19] E. Helfand, H. L. Frisch, and J. L. Lebowitz, J. Chem. Phys. 34, 1037 (1961).

[20] D. Henderson, Mol. Phys. 30, 971 (1975).

[21] S. B. Yuste and A. Santos, Phys. Rev. A 43, 5418 (1991).

[22] S. B. Yuste, M. López de Haro, and A. Santos, Phys. Rev. E 53, 4820 (1996).

[23] M. López de Haro, S. B. Yuste, and A. Santos, in Theory and Simulation of Hard-Sphere Fluids and Related Systems, Lecture Notes in Physics, Vol. 753, edited by A. Mulero (Springer-Verlag, Berlin, 2008) pp. 183-245.

[24] (2016), See Supplemental Material at http://link.aps. org/supplemental/10.1103/PhysRevE.93.062126 for a Mathematica code with all the expressions of this work.

[25] M. Baus and J. L. Colot, Phys. Rev. A 36, 3912 (1987).

[26] M. Abramowitz and I. A. Stegun, eds., Handbook of Mathematical Functions (Dover, New York, 1972).

[27] F. W. J. Olver, D. W. Lozier, R. F. Boisvert, and C. W. Clark, eds., NIST Handbook of Mathematical Functions

tures(Cambridge University Press, New York, 2010).

[28] N. Clisby and B. M. McCoy, J. Stat. Phys. 114, 1343 (2004).

[29] I. Lyberg, J. Stat. Phys. 119, 747 (2005). 
[30] (2016), M. Heinen, private communication.

[31] M. Heinen, E. Allahyarov, and H. Löwen, J. Comput. Chem. 35, 275 (2014).

[32] J. L. Lebowitz, Phys. Rev. 133, A895 (1964).

[33] C. Freasier and D. J. Isbister, Mol. Phys. 42, 927 (1981).

[34] E. Leutheusser, Physica A 127, 667 (1984).

[35] R. D. Rohrmann and A. Santos, Phys. Rev. E 76, 051202 (2007).

[36] S. B. Yuste and A. Santos, J. Stat. Phys. 72, 703 (1993).

[37] A. Santos, (2012), "Radial Distribution Function for Sticky Hard Rods", Wolfram Demonstra- tions Project, http://demonstrations.wolfram.com/ RadialDistributionFunctionForStickyHardRods/.

[38] R. J. Baxter, J. Chem. Phys. 49, 2770 (1968).

[39] B. Barboy, J. Chem. Phys. 61, 3194 (1974).

[40] P. Perry and G. J. Throop, J. Chem. Phys. 57, 1827 (1972), Notice that the numerical values in the two last columns of Table I of this reference are not correct.

[41] Y. Tago and W. R. Smith, Can. J. Phys. 55, 761 (1977). 